\title{
Relative antagonism of mutants of the CGRP receptor extracellular loop 2 domain (ECL2) using a truncated competitive antagonist (CGRP8-37): Evidence for the dual involvement of ECL2 in the two- domain binding model.
}

\author{
Michael J. Woolley ${ }^{1}$, John Simms ${ }^{2}$, Sifat Uddin ${ }^{1}$, David R. Poyner ${ }^{2 *}$ and Alex C. Conner ${ }^{1 *}$ \\ ${ }^{1}$ College of Medical and Dental Sciences, University of Birmingham, Edgbaston, Birmingham, UK. ${ }^{2}$ School of \\ Life and Health Sciences, Aston University, Aston Triangle, Birmingham, UK. \begin{abstract}
Sciences, University of Birmingham, Edgbaston, Birmingham, B15 2TT, UK. d.r.poyner@ aston.ac.uk. School of Life and Health Sciences, Aston University, Birmingham, B4 7ET. The manuscript was written through contributions of all authors.
\end{abstract} \\ * Corresponding authors to whom correspondence should be addressed. Email a.c.conner@bham.ac.uk. College of Medical and Dental
}

\begin{abstract}
The second extracellular loop (ECL2) of the G protein-coupled receptor (GPCR) family is important for ligand-interaction and drug discovery. ECL2 of the family B cardioprotective calcitonin gene related peptide (CGRP) receptor is required for cell signalling. Family B GPCR ligands have two regions; the N-terminus mediates receptor activation and the remainder confers highaffinity binding. Comparing antagonism of $\mathrm{CGRP}_{8-37}$ at a number of point mutations of ECL2 of the CGRP receptor, we show that the ECL2 potentially facilitates interaction with up to the $18 \mathrm{~N}$-terminal residues of CGRP. This has implications for understanding family B GPCR activation and for drug design at the CGRP receptor
\end{abstract}

More than a third of all therapeutic compounds target members of the G protein-coupled receptor (GPCR) superfamily. Understanding how ligands bind to GPCRs is therefore key to modern molecular pharmacology. The second extracellular loop (ECL2) of the GPCR super-family is the largest and most structurally diverse of the ECLs and is vital for ligand binding and activation for almost all of these receptors [1-3]. Crystal structures of the largest sub-family of GPCRs (family A) show ECL2 in a variety of conformations, including beta-sheets, alpha helices and unstructured loops [4-6]. Family B GPCRs includes a smaller group of pharmaceutically important peptide-binding receptors. Conversely, the three available crystal structures of family B GPCRs suggest that ECL2 has no significant secondary structural components [7-9]. Despite this, every biochemical analysis done on the family B GPCR ECL2 regions have found it to be vital for the binding of the respective peptide ligands and the subsequent activation of various signalling pathways [10-12].
Family B GPCRs are all activated by relatively small peptide ligands (typically under 100 amino acids long). They have a diverse range of physiological effects (such as cardioprotection, bone resorption, migraine, digestion control, glucose homeostasis and steroid hormone responses), which has made them of considerable therapeutic interest [13]. These peptide ligands are thought to follow a broadly consistent binding mechanism, termed the two-domain model [14]. Briefly, the C-terminus of the ligand binds to the large extracellular N-terminus of its GPCR. This facilitates the binding of the $\mathrm{N}$-terminus of the ligand to the ECL regions and transmembrane (TM) domain of the receptor. This in turn causes receptor activation and second messenger signalling. It is not known exactly where the ligand $\mathrm{N}$ terminus binds or how that interaction results in the conformational changes that stabilise receptor activation. This information is needed for our understanding of ligand-binding and to provide a platform for the design of specific molecules for individual receptor structures.

One important member of the family B GPCRs is the CGRP receptor. This belongs to the calcitonin-like subfamily and is of considerable therapeutic interest due to the highly potent vasodilatory properties of the CGRP neuropeptide ligand. CGRP has cardioprotective properties of potential beneficial use in heart failure and hypertension $[15,16]$ and has been implicated in the induction of migraine [17]. The CGRP receptor has also been studied extensively because of another unusual feature; it functions as an obligate heterodimer formed by a GPCR subunit (calci- 
tonin receptor-like receptor; CLR) and a single TM spanning protein called receptor activity modifying protein 1 (RAMP 1) [18].

In our recent alanine scan of the CGRP receptor, we substituted 24 residues of the ECL2 region and found that 14 had significant differences in receptor signalling [12]. The largest differences occurred with mutation of residues in the Nterminal half of the loop, although the supporting in silico modelling data predicted the involvement of C-terminal residues in receptor-ligand interactions. This alanine-substitution analysis identified residues required for CGRP receptor function providing a platform for a more mechanistic analysis of ECL2. In the present study, six key residues identified from the alanine scan of the CGRP receptor were selected to investigate the affinity of a truncated version of CGRP missing the first seven residues $\left(\mathrm{CGRP}_{8-37}\right)$. $\mathrm{CGRP}_{8-}$ 37 acts as a reversible, competitive antagonist with an affinity approximately 10-fold lower than full length CGRP. It is thought that CGRP adheres to the two-domain binding model whereby the 8-37 region of CGRP has a discrete binding region that facilitates a second binding event allowing the first seven residues of CGRP to act as the activation domain [14].

Specifically, we are asking whether the ability of $\mathrm{CGRP}_{8-37}$ to antagonise a mutant receptor (where the interaction with the full length CGRP agonist is impaired) is reduced to the same degree as the potency of CGRP. If this is the case, then it would imply that the mutated residue interacted with

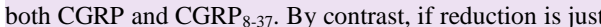
limited to CGRP, it follows that the mutated residue is interacting with only the first seven residues of CGRP (either directly or by affecting a CGRP-independent activation process of the receptor). As labelled $\mathrm{CGRP}_{8-37}$ is not readily available to allow a direct measure of its affinity, this is assessed indirectly by looking at the shift the antagonist causes in the concentration-response curve to CGRP. If the same parallel rightward shift is observed in both WT and mutant receptors, then the affinity and mode of antagonism is also the same.

For this study, we have targeted receptor mutations that either had the biggest reduction in cAMP signalling (R274A, D280A and W283A) or those that were predicted to make ligand contacts in our earlier study (I284A, D287A and T288A) [12].

The binding affinity, expressed as $-\mathrm{Log} \mathrm{Kd}(\mathrm{pKd})$ for $\mathrm{CGRP}_{8-37}$ to the WT and mutant receptors was estimated either by the shift in the dose ratio where there was no significant change in maximum response (Emax) in the presence of the antagonist $\left(\mathrm{pKd}=\left(-\log \left(\left[\mathrm{CGRP}_{8-37}\right] /\right.\right.\right.$ Dose-ratio -1$)$ or by the method of Gaddum where the Emax was depressed [19]. Cos 7 cells were transiently transfected with the WT or mutant receptor construct in a mammalian expression vector pcDNA3.1- (Invitrogen, UK) and co-transfected with a pcDNA3/RAMP1 construct as described previously [12]. Cells were stimulated with CGRP agonist (over a concentration range of $10^{-12} \mathrm{M}$ to $10^{-5} \mathrm{M}$ ) in the presence or absence of $10^{-7} \mathrm{M} \mathrm{CGRP}_{8-37}$ antagonist and cAMP was measured using the FRET-based Lance-assay as described [20]. Isobutyl methyl xanthine (IBMX) was normally present at $1 \mathrm{mM}$ to prevent cAMP breakdown except for kinetic experiments. $10^{-7} \mathrm{M} \mathrm{CGRP}_{8-37}$ was used as lower concentrations of $\mathrm{CGRP}_{8-37}$ gave CGRP activation curves that were poorly resolved from the control in the absence of antagonist; higher concentrations gave incomplete curves (data not shown).

Homology models of the active CLR were generated using Modeller [21] utilising the recently solved cryo-EM structures of the activated Glucagon-like peptide-1 receptor and calcitonin receptor (PDB 5VAI and 5UZ7, respectively) $[22,23] .1000$ models were generated which were subsequently refined and ranked using the membrane relax module of Rosetta [24]. The best model was chosen based on the Rosetta membrane scoring function [24].

The signalling curves for this study are shown in figure 1 . The Emax values of the alanine substitution receptor $(+$ $\mathrm{CGRP}_{8-37}$ ) were compared to a normalised alanine substitution receptor - $\mathrm{CGRP}_{8-37}$ (set to $100 \%$ ) and compared using a one sample t test (table 1 and figure 2). The computed pKd values are shown in table 1 .

I284A resulted in a significant reduction in the affinity of CGRP $_{8-37}$ (table 1). It is likely therefore that the reduction in cAMP signalling observed with the I284A mutant [12] is caused by a disruption of the receptor's ability to interact with the CGRP 8-37 region. There was also a reduction in the affinity for W283A. Conversely, the shift in the CGRP concentration-response curve caused by the CGRP 8 -37 antagonist at the R274A, D280A, and T288A receptors was not significantly different to that seen for the WT CGRP receptor. This suggests that the deleterious effects on signalling of these mutants previously seen [12] is not mediated by the CGRP 8-37 region and therefore, stabilises direct or indirect interactions between the CGRP receptor and the first seven residues of CGRP (the activation domain). However, for all of these mutants as well as W283A, CGRP ${ }_{8-37}$ appeared to act non-competitively at the mutants, with significant reductions in Emax (Figure 2). D287A had no significant effect on CGRP potency, in contrast to our previous study where there was a 7.6-fold reduction in potency [12]. This may reflect cell line variability.

The apparent change in mode of antagonism is unexpected. Caution is needed with this interpretation, as it was not possible to construct full concentration-response curves to CGRP in the presence of $\mathrm{CGRP}_{8-37}$ for the mutants with lower CGRP potency, as the required concentrations of CGRP would be impractical. However, when we fit "expected" concentration-response curves in accordance with competitive inhibition assuming a full Emax (dotted line graphs in figure 1), then the shift in the curve for CGRP for the mutant receptors would be even greater than for the WT receptors; i.e. the affinity for $\mathrm{CGRP}_{8-37}$ would have increased. In either interpretation, these mutations give unexpected effects.
Commented [MW1]: Is this wording definitely correct? I found myself having to re-read it a few times to try and be clear. I wasn't sure if we said the same thing for the two different examples or not! I think this is the hardest bit to explain of the paper and I'm not sure how to improve it. 
There was no evidence of any non-competitive inhibition by CGRP $_{8-37}$ on the WT receptor, even when cells were transfected with only $10 \%$ of the normal CLR/RAMP1 cDNA (data not shown). Under these conditions, any receptor reserve will have been removed (the maximum stimulation of cAMP was reduced by $93 \%$ ), which might otherwise mask non-competitive behaviour of antagonists. There was still a clear shift of the CGRP concentration response curve in the presence of $10^{-7} \mathrm{M} \mathrm{CGRP}_{8-37}$ (shift $1.54 \pm 0.35, \mathrm{n}=3$ ), but there was no significant change in the Emax in the presence of the antagonist ( $125 \pm 25 \%$ of CGRP alone).

Of the residues examined in this study, only I 284 is predicted to face bound CGRP in a model of CLR (figure 3); interestingly it interacts with the 8-18 helix of the peptide.

T288 may have a weak interaction with the extreme N-terminus of CGRP, but the other residues face into the binding pocket. The model is consistent with cryo-electron microscope structures showing the binding of calcitonin and GLP-1 to their receptors [22, 23]. In the GLP-1 receptor (5VAI at the Protein Structure Databank) the equivalent of I284, T298, is adjacent to S18 of GLP-1. The model suggests it is unlikely that any part of ECL2 interacts with the extracellular domain of CLR of RAMP1, unless the signal peptide of CLR is not cleaved. We speculate that the R274, D280 and W283 and (to a lesser extent) T288 are important for maintaining the architecture of the peptide-binding pocket. In support of this, there is a correlation between the reduction in CGRP potency at the mutants and the decrease in Emax in the presence of CGRP $_{8-37}$ (figure 2b). We suggest that when R274, D280 and W283 and T288 are mutated, residues 1-7 of CGRP no longer fit easily into this pocket, whereas $\mathrm{CGRP}_{8-37}$ binds relatively unimpaired. To test this, we investigated the time course of cAMP production by CGRP at W283A in the absence of IBMX, normally present in our assays to prevent cAMP breakdown. This confirmed that the kinetics of receptor activation was much slower in this mutant compared to WT, consistent with a reduction in the association rate of binding of CGRP. By contrast the kinetics of CGRP at I284A are the same as WT (figure 2c).

This work is the first mutational evidence to map the interaction of CGRP within ECL2 of its receptor. The data suggests that the mode of interaction of $\mathrm{CGRP}_{8-37}$ with the

CGRP receptor is likely to be more complicated than predicted from a simple consideration of the two-domain model [14] where residues 1-7 are considered to be the sole determinant of agonist activity. In a previous structure-activity study, we demonstrated that mutations to residues 8 and 9 of CGRP gave partial agonists, challenging a simplistic notion that $\mathrm{CGRP}_{1-7}$ is the sole determinant of receptor activity [25]. This is further supported by a study on the allied calcitonin receptor, where $\mathrm{CGRP}_{8-37}$ is a partial agonist at the complex between this receptor and RAMP1 [26]. The current data suggests that a number of residues of CGRP beyond the first seven interact in the vicinity of ECL2 and so could directly influence the TM bundle. This helps our understanding of receptor activation as well as emphasising the key role of ECL2 in the CGRP receptor.
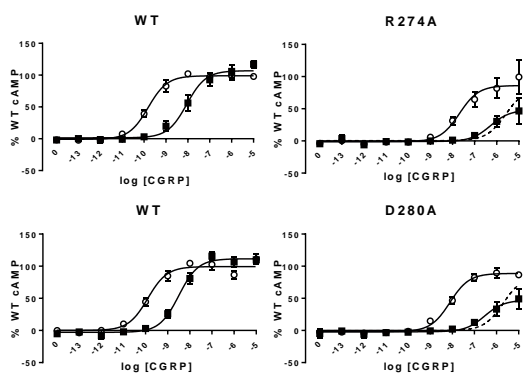

W 283A

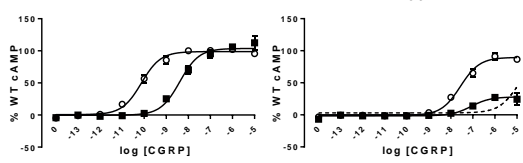

$1284 \mathrm{~A}$

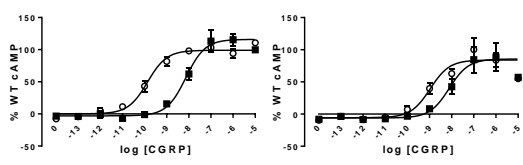

D 287 A

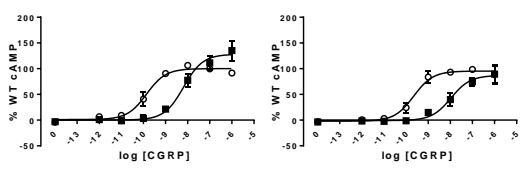

wT

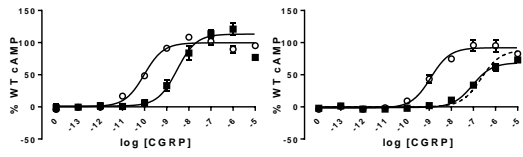

Figure 1. Concentration-response curves to CGRP of Cos7 cells transfected with WT CGRP receptor (WT CLR + R1) or alanine substitution receptor (mutant CLR + R1). Cells were stimulated with CGRP in either the presence (open circles) or absence (closed circles) of $10^{-7} \mathrm{M} \mathrm{CGRP}_{8-37}$. Raw cAMP values were normalized to WT (in the absence of CGRP $_{8-37}$ ) basal and Emax values. The curves show means \pm s.e.m values of 3-5 determinations. The broken lines in the graphs of the mutant receptors show the fit if it is assumed that the maximum response is unchanged in the presence of $\mathrm{CGRP}_{8-37}$ 

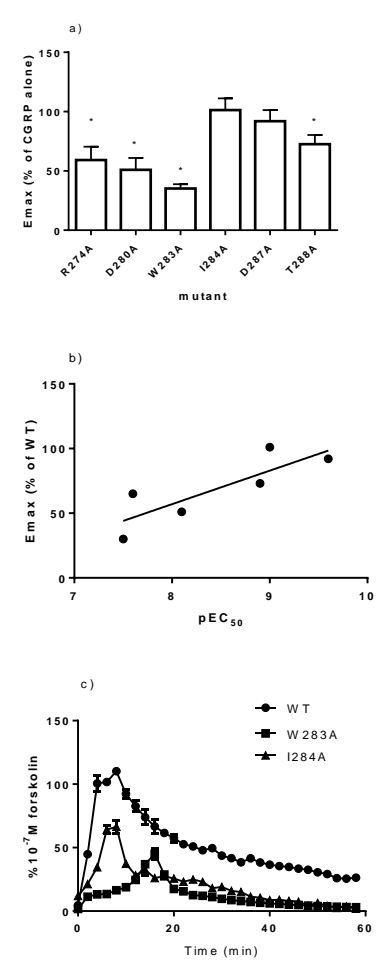

Figure 2a. \% Emax values of mutants in the presence of $\mathrm{CGRP}_{8-37}$ relative to Emax values without antagonist. Values are means \pm s.e.m. of 3-7 determinations. Significant differences compared to 100 were determined using a one sample $\mathrm{t}$ test, ${ }^{*} \mathrm{p}$ value $<0.05$. b) Correlation between $\mathrm{pEC}_{50}$ for CGRP and change in Emax at alanine mutants. c) Time course of cAMP production in response to CGRP at WT, W283A and I284A receptors. Points are means \pm s.e.m. of 3 determinations.

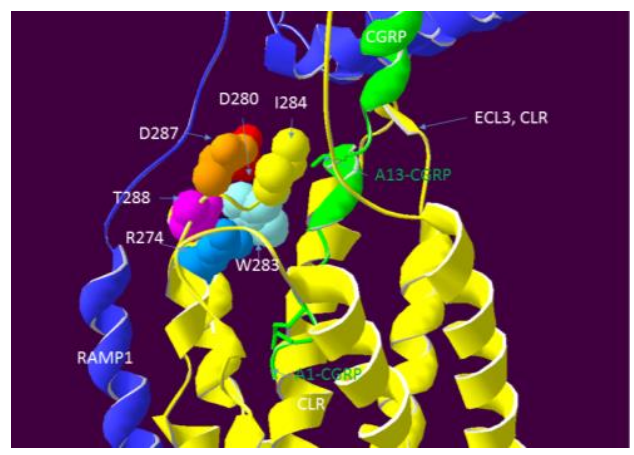

Figure 3. Model of CGRP (green) bound to CLR (yellow) and RAMP1 (blue), showing key residues in ECL2 of CLR.
Table 1. Estimated pKd values for $\mathrm{CGRP}_{8-37}$ at WT and mutant CGRP receptors

\begin{tabular}{|c|c|c|c|}
\hline CLR Mutant & $\mathrm{pKd}$ WT & $\mathrm{pKd}$ mutant & $\mathrm{n}$ \\
\hline $\mathrm{R} 274 \mathrm{~A}$ & $8.64 \pm 0.10$ & $8.40 \pm 0.05$ & 4 \\
\hline $\mathrm{D} 280 \mathrm{~A}$ & $8.41 \pm 0.01$ & $8.43 \pm 0.15$ & 4 \\
\hline $\mathrm{W} 283 \mathrm{~A}$ & $8.67 \pm 0.07$ & $7.51 \pm 0.19^{* * *}$ & 7 \\
\hline $\mathrm{I} 284 \mathrm{~A}$ & $8.77 \pm 0.09$ & $7.85 \pm 0.12^{* * *}$ & 4 \\
\hline $\mathrm{D} 287 \mathrm{~A}$ & $8.79 \pm 0.33$ & $8.65 \pm 0.35$ & 3 \\
\hline T288A & $8.54 \pm 0.18$ & $8.87 \pm 0.21$ & 4 \\
\hline
\end{tabular}

Values are means \pm s.e.m. $* * *$ p value $<0.001$ versus WT (unpaired Student's t-test).

\section{ASSOCIATED CONTENT}

\section{Funding Sources}

Research funded by the British Heart Foundation (BHF), grant number [PG/12/59/29795] and the BBSRC [BB/M007529/1]. 
Notes

The authors declare no competing financial interests.

\section{ACKNOWLEDGMENT}

We thank Chris Reynolds for useful discussions

\section{ABBREVIATIONS}

(Word Style "Section_Content"). CLR, calcitonin receptor-like receptor; CGRP, calcitonin gene related peptide; ECL, extracellular loop GPCR, G protein-coupled receptor; TM. transmembrane; WT, wild-type.

\section{REFERENCES}

1. Palczewski K, Kumasaka T, Hori T, Behnke CA, Motoshima H, et al. (2000) Crystal structure of rhodopsin: A G protein-coupled receptor. Science 289: 739-745.

2. Cherezov V, Rosenbaum DM, Hanson MA, Rasmussen SG, Thian FS, et al. (2007) High-resolution crystal structure of an engineered human beta2 adrenergic G protein-coupled receptor. Science 318: 1258-1265.

3. Chien EY, Liu W, Zhao Q, Katritch V, Han GW, et al. (2010) Structure of the human dopamine D3 receptor in complex with a D2/D3 selective antagonist. Science 330: 1091-1095.

4. Wu H, Wacker D, Mileni M, Katritch V, Han GW, et al. (2012) Structure of the human kappa-opioid receptor in complex with JDTic. Nature 485 327-332.

5. Haga K, Kruse AC, Asada H, Yurugi-Kobayashi T, Shiroishi M, et al. (2012) Structure of the human M2 muscarinic acetylcholine receptor bound to an antagonist. Nature 482: 547-551.

6. Warne T, Serrano-Vega MJ, Baker JG, Moukhametzianov R, Edwards PC, et al. (2008) Structure of a beta1-adrenergic G-protein-coupled receptor. Nature 454: 486-491.

7. Hollenstein K, Kean J, Bortolato A, Cheng RK, Dore AS, et al. (2013) Structure of class B GPCR corticotropin-releasing factor receptor 1. Natur 499: 438-443.

8. Siu FY, He M, de Graaf C, Han GW, Yang D, et al. (2013) Structure of the human glucagon class B G-protein-coupled receptor. Nature 499: 444449.
9. Jazayeri A, Dore AS, Lamb D, Krishnamurthy H, Southall SM, et al. (2016) Extra-helical binding site of a glucagon receptor antagonist. Nature 533: 274-277.

10. Koole C, Wootten D, Simms J, Miller LJ, Christopoulos A, et al. (2012) Second extracellular loop of human glucagon-like peptide-1 receptor (GLP1R) has a critical role in GLP-1 peptide binding and receptor activation. Biol Chem 287: 3642-3658.

11. Gkountelias K, Tselios T, Venihaki M, Deraos G, Lazaridis I, et al. (2009) Alanine scanning mutagenesis of the second extracellular loop of type 1 corticotropin-releasing factor receptor revealed residues critical for peptide binding. Mol Pharmacol 75: 793-800.

12. Woolley MJ, Watkins HA, Taddese B, Karakullukcu ZG, Barwell J, et al. (2013) The role of ECL2 in CGRP receptor activation: a combined modelling and experimental approach. J R Soc Interface 10: 20130589.

13. Hollenstein K, de Graaf C, Bortolato A, Wang MW, Marshall FH, et al. (2014) Insights into the structure of class B GPCRs. Trends Pharmacol Sc 35: $12-22$.

14. Hoare SR (2005) Mechanisms of peptide and nonpeptide ligand binding to Class B G-protein-coupled receptors. Drug Discov Today 10: 417-427.

15. Brain SD, Grant AD (2004) Vascular actions of calcitonin gene-related peptide and adrenomedullin. Physiol Rev 84: 903-934.

16. Smillie SJ, Brain SD (2011) Calcitonin gene-related peptide (CGRP) and its role in hypertension. Neuropeptides 45: 93-104.

17. Russell FA, King R, Smillie SJ, Kodji X, Brain SD (2014) Calcitonin gene-related peptide: physiology and pathophysiology. Physiol Rev 94 1099-1142.

18. McLatchie LM, Fraser NJ, Main MJ, Wise A, Brown J, et al. (1998) RAMPs regulate the transport and ligand specificity of the calcitonin-receptor-like receptor. Nature 393: 333-339.

19. Kenakin TP (2014) A Pharmacology Primer: Techniques for more ef fective and strategic drug discovery ( $4^{\text {th }}$ Edition): Academic Press, Elsevier. Section 6.4.

20. Hunter MR, Glass M (2015) Increasing the flexibility of the LANCE cAMP detection kit. J Pharmacol Toxicol Methods 71: 42-45.

21. Hay DL, Harris PW, Kowalczyk R, Brimble MA, Rathbone DL, et al. (2014) Structure-activity relationships of the N-terminus of calcitonin generelated peptide: key roles of alanine- 5 and threonine- 6 in receptor activation. Br J Pharmacol 171: 415-426. 
Authors are required to submit a graphic entry for the Table of Contents (TOC) that, in conjunction with the manuscript title, should give the reader a representative idea of one of the following: A key structure, reaction, equation, concept, or theorem, etc., that is discussed in the manuscript. Consult the journal's Instructions for Authors for TOC graphic specifications.

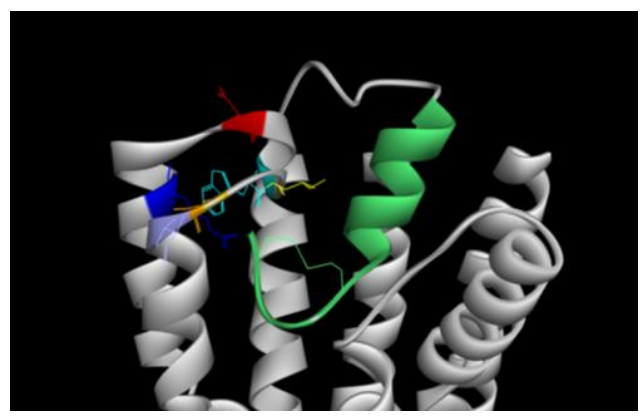

